\title{
The use of time and trajectory forms as visual information about spatial scale in events
}

\author{
DANIEL S. MCCONNELL, MICHAEL M. MUCHISKY, and GEOFFREY P. BINGHAM \\ Indiana University, Bloomington, Indiana
}

\begin{abstract}
Spatial metrics are lost but temporal metrics are preserved in the mapping from events to optic flow. In inanimate events governed by gravity, temporal scale is linked to spatial scale in ways specific to particular events. We tested whether time can be used as information about spatial scale in visually recognizable events. On average, observers were able to judge object size in event displays that eliminated information other than time and trajectory forms. However, judgment variability was substantial. After feedback on one event, observers judging distance performed better and generalized training to other events. Observers are sensitive to the general form of the scaling relation, but they require feedback to attune event-specific constants.
\end{abstract}

The problem of size perception is that image size varies with the distance of an object. A traditional solution has been to appeal to the apprehension of distance to derive perception of size. However, the overarching problem common to both size and distance perception is that definite spatial metrics are lost in the mapping from objects and events into optical pattern. Optical patterns are characterized instead by gradients of image size, of optical texture density, or of optical velocities (i.e., motion parallax). Judging from our ability to scale actions (e.g., reaching or throwing) appropriately, we must nevertheless be able to perceive definite spatial scale visually. How is this possible?

Familiar size is a potential solution to the size perception problem that is independent of distance perception (Epstein, 1967). The observer is assumed to know or remember from previous experience the definite size of familiar objects. This solution is appropriate for recognizable objects that have a highly reliable and restricted range of sizes. Using familiar size, an observer could, in principle, derive the distance of an object from its visual angle. Of course, the observer must first recognize the object, which he or she achieves by identifying its characteristic form. Thus, size and distance perception is reduced to form perception. Bingham (1993a) explored this idea in the context of the perception of the size of biological objects (trees). Although the size of trees varies widely, tree forms vary with their height, raising the possibility that observers might use tree form as information about height. Physical scaling laws constrain trunk diameter and the number of branches to vary systematically in proportion to tree height. Using these relations, Bingham (1993a) created

The authors thank Christopher Pagano and Michael Stassen for helpful discussion, Chong Woo Kim and Chad Stafford for help in collecting the data, and Heiko Hecht, Sverker Runeson, and Hal Sedgwick for very thorough and helpful reviews. Correspondence should be addressed to D. S. McConnell, Department of Psychology, Indiana University, Bloomington, IN 47405 (e-mail: dsmcconn@indiana.edu). displays of trees of different simulated heights, with the image height held constant. Observers were found to be able to use variations in form as information about tree height (Bingham, 1993a).

Visual information about scale has also been explored in the context of event perception. Runeson (1977b) proposed that kinematic patterns can lawfully specify the dynamical properties of an event-a possibility subsequently termed kinematic specification of dynamics (KSD) (Runeson, 1994; Runeson \& Frykholm, 1983). Observers can detect and use kinematics as information about the scale of mass-related properties in events, as shown, for example, by studies on the perception of the relative mass of two colliding balls (Runeson \& Vedeler, 1993; Todd \& Warren, 1982) and the perception of lifted weight (Bingham, 1987, 1993b; Runeson \& Frykholm, 1981).

KSD as such is about the perception of dynamic (i.e., mass-related) properties. Pittenger $(1985,1990)$ generalized the idea to the perception of the scale geometric properties in events. The law of pendulum motion relates the length of a simple pendulum to its period of motion. Potentially, observers could judge the length of a pendulum given information about its period. Pittenger (1985) found that observers could do this, but they produced increasing underestimation with increasing length. When viewing normal and perturbed pendulums, however, observers could rate the naturalness of motion to a high degree of accuracy (Pittenger, 1990).

The idea has also been applied to the perception of the distance of objects in free fall (Saxberg, 1987a, 1987b; Watson, Banks, von Hofsten, \& Royden, 1992). The law of free fall motion relates the height of fall to the duration of the event; thus, observers could potentially use this as information about size or distance. Saxberg (1987b) tested this in a simulated catching task, in which the ball approached the observer along a parabolic trajectory. The optic flow projected from the event included both image expansion from approach and a vertical component of free fall. Although observers performed this task well, they per- 
formed poorly in a condition in which image size was held constant. Saxberg (1987b) argued that the latter instance isolated free fall as the sole source of information in the display, and the subsequent failure of observers to catch the ball demonstrated their lack of ability to use this information. However, Watson et al. (1992) argued that the display entailed conflicting sources of information. Trajectory information specified motion toward the observer, but the static image size specified a constant distance from the observer.

Recently, Hecht, Kaiser, and Banks (1996) investigated whether observers can estimate size and distance in displays of simulated balls in free fall. Their displays contained only the vertical motion of a ball in the frontoparallel plane. Observers were successful in estimating scale roughly, but performance was less than optimal. The authors concluded that the information is not well used.

Finally, using an approach similar to that used by Pittenger (1990), Stappers and Waller (1993) have reported that observers can adjust the gravitational constant in computer simulations to make the motion of falling objects appear natural.

The essence of the solutions above (Pittenger, 1985; Saxberg, 1987a; Watson et al., 1992) is that the spatial scale, $s$, of a recognizable event is linked to the temporal scale, $t$, via a natural law. The general form of this relation for these gravitationally governed events is

$$
s=k t^{2},
$$

where $k$ is a constant specific to the event being considered. This relation is an ideal description that ignores the effects of friction and air resistance. However, we note for future reference that, in actual events, these forces affect an object's trajectory as a function of mass, density, velocity, surface structure, and so on. Assuming motion in the frontoparallel plane, the definite distance of fall of a sphere in free fall is specified as

$$
s=(g / 2) t^{2},
$$

where $\mathrm{g}$ is the gravitational constant of acceleration $\left(9.81 \mathrm{~m} / \mathrm{sec}^{2}\right)$. For a sphere rolling down a slope, without slipping, the distance along the slope is

$$
s=(5 g / 7) \sin (\alpha) t^{2}
$$

where $\alpha$ is the angle of the slope from the horizontal. Finally, for a simple pendulum, the length of the pendulum is

$$
s=\left(g / 4 \pi^{2}\right) t^{2},
$$

where $t$ in this case is the period of the pendulum. The only difference among these relations is the value of an eventspecific constant, $k$. Thus, once generalized across different events, this solution becomes an extension of familiar size as treated by Bingham (1993a), where recognition yields not scale directly but the correct relation to use as information about scale. Here, the characteristic form to be recognized is an oriented trajectory form (e.g., free fall or pendular motion) instead of an object form (e.g., a tree) (Bingham, 1995; Bingham, Rosenblum, \& Schmidt,
1995). In each instance, a lawful relation scales an eventspecific length (e.g., height of fall or length of pendulum) to an event duration. When the event-specific constant incorporates the reciprocal of the visual angle of the relevant length in the event, duration yields information about the distance to the event. By taking a ratio of the visual angles of this length and some other object in the event, duration can also yield information about that object's size.

Can observers judge scale in these events given only temporal information? Previous work has suggested that they might (Pittenger, 1985), but the judgments were rather inaccurate and imprecise. Furthermore, each of the previous studies has examined only one type of event at a time, so that spatial scale has been confounded with event duration (i.e., they have covaried uniquely). Were observers judging spatial scale or merely judging time? Muchisky and Bingham (1992) separated these two variables by presenting observers with computer simulations of a number of different events. Because the scaling constant is different in each type of event, durations corresponding to a given actual size varied widely. Observers judged scale in the events at about the same level of accuracy as that found by Pittenger (1985) and Hecht et al. (1996). If observers had relied on duration to estimate size, performance would have varied across events as a function of the differences in event-specific scaling coefficients. It did not. Accordingly, Muchisky and Bingham concluded that observers were judging spatial scale and not merely event duration.

\section{EXPERIMENT 1}

In Experiment 1, we extended Muchisky and Bingham's (1992) approach by presenting observers with a wider array of events. Observers were presented with displays depicting balls in free fall, balls free falling and then bouncing on a hard surface, balls rolling down an incline. and balls swinging at the end of a pendulum. To further decouple actual size from event duration, we included inclines at three different angles and pendulums of three different lengths. The question was, Can observers use trajectory information to perceive the size of these balls, given constant image size and no other information about scale? Finally, observers in Muchisky and Bingham's study had a limited response range to choose from when making judgments. It is possible that the observers parsed the response range into categories and placed balls within these categories. To control for this potential bias, we divided our observers into two groups, each with a different response range.

\section{Method}

Observers. Twenty-one graduate and undergraduate students at Indiana University participated in the study. All had normal or corrected-to-normal vision. The observers were paid either $\$ 4.25$ or $\$ 5$ per hour.

Display generation. Balls of six sizes were simulated in eight different events. Balls appeared as simple black line drawings on a 
white screen. Also, a black line was drawn on each ball as a radius, so that the rotational motion of the ball could be seen. Quicktime movies (compressed digital movies) of simple planar events were created using event-dynamic models generated by the application Interactive Physics II (1992), which has built-in algorithms for generating simulations of moving objects and collisions between them. These models included components for earth-normal gravity and air resistance. A numerical algorithm calculated position at successive time intervals $(\Delta t=.013)$. The application MacroMind Accelerator (1989) was used to lock the frame rate of the simulations to the frame rate of the monitor, which was $75 \mathrm{~Hz}$. The result was smooth and continuous motion. The six simulated ball sizes were $2.54,10.16,20.32,30.48,40.64$, and $60.96 \mathrm{~cm}$. The balls were simulated to have the density and elasticity of hard wood.

We used simulated distances and heights appropriate for parallel projection in the frontoparallel plane. The image sizes of the balls were held constant across all events and simulated actual sizes. This was accomplished by covarying simulated distance from the projection surface (the screen) with simulated size. The displays were designed so that there was no information about scale apart from variations in trajectories. For each display, the ball began in a resting position and then immediately started moving.

Free fall (FF) was the first type of event simulated. A ball in FF appeared near the top of the display, fell, and disappeared near the bottom of the display. In a variation of the FF event, the ball fell and bounced several times on a simulated wooden platform near the bottom of the screen (free fall and bounce, FFB). The platform appeared as a rectangular outline.

The second type of event was a ball rolling without slipping down an incline, from left to right across the screen. The incline appeared as a triangular outline. The third type of event was a pendulum. The ball was the bob of the pendulum. In this event, the pendulum was shown swinging back and forth for two cycles. In all analyses, the duration of the pendulum was defined as the period of one cycle. In the case of the FFB event, duration was defined as the duration of the initial free fall. For all other events, event duration was defined as extending from the initiation of ball movement to the ball's disappearance from the screen.

We also manipulated event duration by using three different slope gradients - shallow (ShS, $\alpha=10^{\circ}$ ), medium (MS, $\alpha=20^{\circ}$ ), and steep (StS, $\alpha=30^{\circ}$ ) -and three different pendulum lengths - short (SP), medium (MP), and long (LP). The SP length on the screen was $2.5 \mathrm{~cm}$, the MP was $5 \mathrm{~cm}$, and the LP was $7.5 \mathrm{~cm}$. Each gradation of the slope or of the pendulum produced a different period for a ball of a given size. If the observers based their judgments on event duration as such, then they should produce systematic errors accordingly.

The combination of six sizes with eight events resulted in 48 different displays. For each trial, the display was repeated three times. Displays were presented to the observers on a Macintosh II, with a $50.8-\mathrm{cm}$ monitor. The observers were seated directly in front of the screen at a distance of about $60 \mathrm{~cm}$.

Procedure. The observers were instructed to judge the definite size of simulated balls and to use the form of the motion as the basis of their judgments. The experimenter described the relation between image size and actual size and described the way that constant image size was achieved by covariation of size and distance. This was illustrated using large and small balls and holding them at distances that preserved image size. The two balls were also used to demonstrate what was meant by the form of motion and how balls of different sizes may vary in their motion. This was accomplished by having the observers view the balls dropped at different heights and distances, so that image size was preserved.

The observers were shown four demonstration trials in order to familiarize them with the displays and the computer. They were then presented with a randomly ordered block of 48 trials. The observers judged size in a manner designed to reduce the problems associated with verbal responses and the explicit use of numbers (see Poulton, 1989, for a discussion). After viewing the three presentations of a given trial, the observer held out his or her hands as if grasping the object between them, and we measured the distance between the hands with a $122-\mathrm{cm}$ ruler attached to the edge of a table in front of the observer. The observer's response range was determined by the length of the tape measure. To control for the potential effect of the size of the response range, we used two ranges. Thirteen observers were given a range up to $122 \mathrm{~cm}$, roughly corresponding to the maximum hand span, and 8 observers were given a range up to $183 \mathrm{~cm}$. (The largest ball size was $60.96 \mathrm{~cm}$.) For the large-range group, the observers were instructed to imagine the bali sitting on the ground next to them and to place their hand at the height of the ball. We measured the height using a vertical ruler marked on a nearby wall.

\section{Results}

The displays used in this experiment were impoverished. Information provided by convergence, disparity, or accommodation was incorrect, and it had to be ignored by the observers. Furthermore, texture gradients, perspective transformations, and other sources of information about depth were missing from the computer simulations. Thus, the observers were required to estimate scale from displays containing minimal and contradictory information. This task was difficult, and, as a result, 6 observers failed to understand what was required of them, and they were excluded from the analysis. This left 10 observers in the 122-cm-range group, and 5 in the 182-cm-range group.

Using the data of all observers in the 122-cm-range group, judged size was regressed against actual size. The result was a slope of .85 , a $y$-intercept of 4.27 , and an $r^{2}$ of .37. This $r^{2}$ reflects a large amount of both between- and within-subjects variability. Individual observers performed at a comparable level, with a mean $r^{2}$ of .47 $(S D=.13)$. The same analysis was performed on the data of the 182-cm-range group, yielding a slope of .77, a $y$ intercept of 8.33, and an $r^{2}$ of .18. Although there was even greater between-subjects variability in this group, the performance of individual observers was similar to that of the other group, with a mean $r^{2}$ of $.33(S D=.20)$. Mean data from each of these two groups are shown in Figure 1. A multiple regression comparing the slopes and intercepts of the two range conditions was significant $\left[F(3,717)=97.94, p<.001, r^{2}=.29\right]$. Only the actual size factor (partial $F=238.6, p<.001$ ) and the intercept factor (partial $F=8.45, p<.005$ ) were significant, indicating that mean judgments in the $182-\mathrm{cm}$ condition were greater by about $4 \mathrm{~cm}$. This difference was small given the $60-\mathrm{cm}$ increase in the size of the range of possible judgments. If mean judgments had increased in proportion to the change in the range, the judgments of the largest size should have increased by $30.5 \mathrm{~cm}$. The actual increase was only $13 \%$ of this.

If the observers were estimating size accurately, there should be no differences between events when judgments are regressed on actual size. That is, judgments should all fall on a single line. If, on the other hand, the observers 


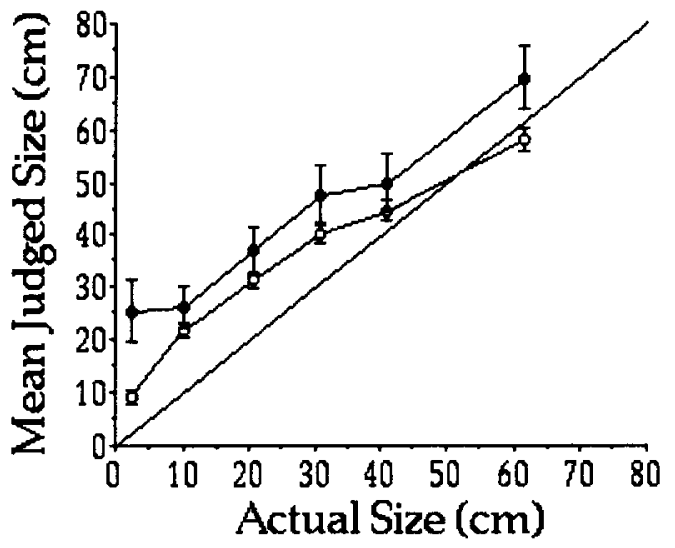

Figure 1. Mean judged size (in centimeters) regressed against actual size (in centimeters) for the 122-cm-range condition (open circles) and the $182-\mathrm{cm}-$-range condition (filled circles), with standard error bars.

were merely judging event duration, then regression of judgment on actual size should yield a fan of lines, each differing in slope, one for each event. This is illustrated in Figure 2, where actual sizes are plotted against event durations for each event. (As predicted by Equations 1-4, these lines should be straight. The slight curvature is due to the effect of friction included in the simulations.) Within a given event, duration and actual size covaried. However, across events, including the different pendulum and slope events, many different durations corresponded to a given actual size. These would have elicited significantly different judgments for a given actual size if the observers had based their judgments only on event duration as such. We performed multiple regressions comparing two events at a time yielding 28 comparisons (i.e., eight events taken two at a time). We combined the data of the two range groups because the increase in judgments as a result of the range manipulation was small. First, we performed an analysis comparing events with respect to the relation between judgments and actual size. As shown in Table 1, we found significant slope differences in 7 of 28 comparisons (i.e., $25 \%$ ). We found no differences in intercepts. Figure 3 shows the mean judgments plotted against actual size for each event. This failed to yield the fan pattern that would be expected if judgments were based solely on event durations.

Next, we compared events with respect to the relation between judgments and event durations. In this instance, 19 of 28 slope comparisons (or $68 \%$ ) were found to be significantly different, as shown in Table 2 . Again, there were no significant differences in intercept. Mean judgments were plotted against event durations for each event (Figure 4). This plot did yield the fan-shaped pattern, indicating that judgments reflected actual size rather than durations.

Finally, the multiple regressions yielded coefficients reflecting the magnitude of the differences in slope. In Tables 1 and 2, we report the magnitudes of these slope dif- ferences in proportion to the overall mean slopes in each case. This allows direct comparison of the slope differences from the two analyses. The mean proportional change in the judgments versus actual distance comparisons was $0.57(S D=0.2)$, whereas that in the judgments versus event duration comparisons was $1.25(S D=0.4)$. So, not only were there more slope differences when judgments were compared with event duration but the magnitude of the differences that did occur was over twice as large.

Taken together these results indicate that the observers were able to use time as information about spatial scale in simple inanimate events. Given our displays, this entailed the ability to use trajectory forms to recognize each type of event (Bingham, 1995; Bingham et al., 1995) so that event timing could be appropriately scaled to yield information about size.

\section{EXPERIMENT 2}

The judgments in Experiment 1 were fairly imprecise. Both within-subjects variability and between-subjects variability were large. This is consistent with results of previous studies. Why should these judgments be so variable? All observers found the task to be difficult. The simulations were presented under reduced and rather unrealistic viewing conditions that entailed contradictory information. The balls were depicted as mere line drawings and, accordingly, appeared only as such. Viewing them as objects at some distance beyond the computer screen required considerable imagination despite the relatively realistic trajectories.

To reduce these problems, we next used displays of real balls moving on real surfaces viewed via a headmounted video display. Patch-light displays were used to isolate motion as information, and ball size was covaried with viewing distance to eliminate variations in image size. In Experiment 1, the observers alternated between viewing outline balls in the display and their own hands in a fully lit room. This transition served to heighten the abstractness and imaginative character of the task. Also, re-

Table 1

Significant Slope Differences in

Multiple Regressions of Actual Size on Judged Size for Events Compared Two at a Time

\begin{tabular}{lcccccccc}
\hline & FF & FFB & ShS & MS & StS & SP & MP & LP \\
\hline FF & - & & & & & & & \\
FFB & & - & $8.0 \dagger$ & & $4.0^{*}$ & & $8.0^{\dagger}$ & $9.9 \dagger$ \\
ShS & & 0.75 & - & $4.3^{*}$ & & & & \\
MS & & & 0.46 & - & & & $4.2^{*}$ & $5.8^{*}$ \\
StS & 0.64 & & & - & & & \\
SP & & & & & & - & & \\
MP & 0.73 & & 0.43 & & & & - \\
LP & 0.80 & & 0.50 & & & & - \\
\hline
\end{tabular}

Note-FF, free fall; FFB, free fall and bounce; ShS, shallow slope; MS, medium slope; StS, steep slope; SP, short pendulum; MP, medium pendulum; LP, long pendulum. Upper triangle: ${ }^{*} p<.05 ;{ }^{+} p<.01$. Lower triangle: differences in slope in proportion to mean slope. 


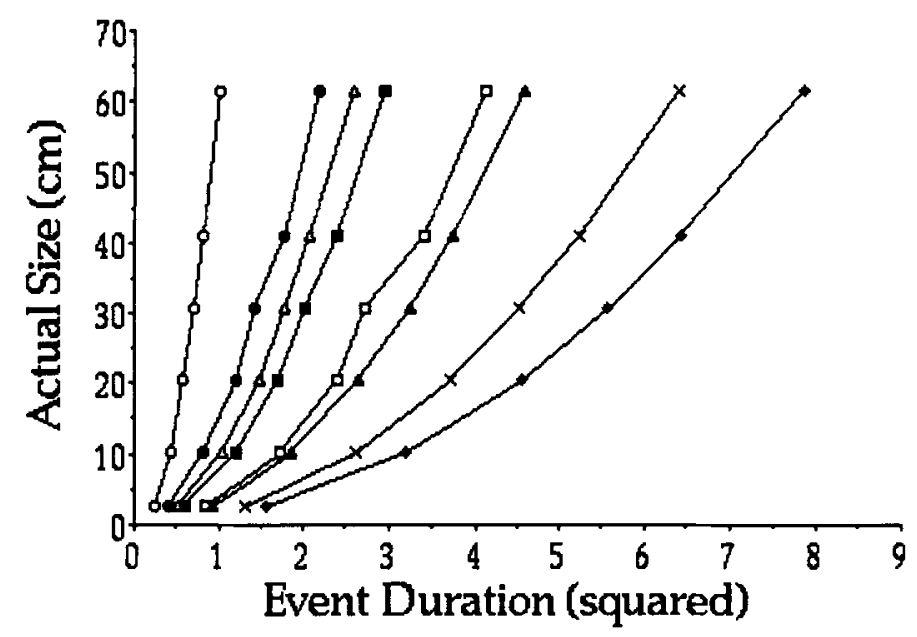

Figure 2. Actual size (in centimeters) regressed against event duration (in seconds) squared for all eight events.

stricting the size of the visual field and viewing through a frame have been found to produce underestimation of size or distance (Bingham \& Pagano, 1998; Eby \& Braunstein, 1995). To avoid the schism between display and response and to control for the viewing conditions, we used the same head-mounted video display in both display and response conditions. This required a change to judgment of distance rather than size. The observers manipulated a pulley to move a patch-light ball to the distance of the patch-light ball observed in the event display. The pulley-mounted ball was observed through a head-mounted camera and video display while the observer moved the pulley with his/her hand.

While the viewing conditions might have been responsible for some of the judgment variability in Experiment 1 , the majority of the variability might have been attributed to a failure of the observers to be well attuned to the precise values of the scaling constants. The results of Experiment 1 suggest that observers are sensitive to the form of the scaling relation between temporal and spatial scale and to the scaling relations among events. Although the observers appear to have maintained the appropriate ratios between the scaling constants for different events, they did not exhibit accurate perception of definite spatial scale. That is, they perceived relative size accurately, but they failed to perceive definite size accurately.

Why might observers not be well attuned to the scaling constants, especially if they are sensitive to the relationships among them? The hypothesized information about scale in these events is approximate. It does not take into account the effects of friction or air resistance and, therefore, of mass. The effects of friction and air resistance vary as a function of object mass, density, and surface structure. For the present purposes, we will focus on the effects of variations in mass density as compared with the effects of viewing distance as illustrated in Figure 5. The trajectories of three balls rolling down an inclined plane are shown in an optical phase portrait - that is, a plot of the tangential velocity and position of the balls' centers of mass as mapped into corresponding optical velocities and positions (Bingham, 1995). The trajectories were generated with the Interactive Physics II (1992) software, simulating a $1-\mathrm{m}$ ball weighing $1 \mathrm{~kg}$, a $0.7-\mathrm{m}$ ball of equal density $\left(1.27 \mathrm{~kg} / \mathrm{m}^{3}\right)$ weighing $0.56 \mathrm{~kg}$, and a $1-\mathrm{m}$ ball of greater density $\left(1.82 \mathrm{~kg} / \mathrm{m}^{3}\right)$ weighing $1.43 \mathrm{~kg}$. In the simulations, air resistance was linear in the tangential velocity, and gravity and air resistance were earth normal. The viewing distance for each ball was such that the image size was the same for all three; so the $0.7-\mathrm{m}$ ball was viewed at a smaller distance than the other two. Consider the $1-\mathrm{m} 1-\mathrm{kg}$ ball as a standard. A decrease in size and viewing distance, while holding density constant, results in a quantitative change in the trajectory-namely, an increase in optical velocities, as expected from Equation 1 . However, an increase in density, while holding size

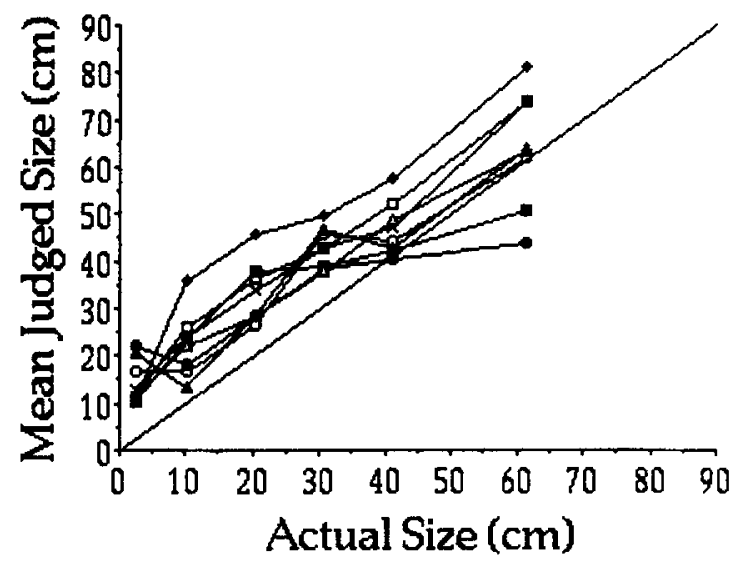

Figure 3. Mean judged size (in centimeters) regressed against actual size (in centimeters) for all eight events: FF (open circles), FFB (filled circles), ShS (open squares), MS (filled squares), StS (open triangles), SP (filled triangles), MP (crosses), and LP (filled diamonds). 
Table 2

Significant Slope Differences in Multiple Regressions of Event Duration on Judged Size for Events Compared Two at a Time

\begin{tabular}{lcccccccr}
\hline & FF & FFB & ShS & MS & StS & SP & MP & LP \\
\hline FF & - & & $17.1_{\ddagger}^{\ddagger}$ & $18.2_{\ddagger}^{\ddagger}$ & $18.3 \ddagger$ & $21.9 \ddagger$ & $24.8 \ddagger$ & $21.3 \ddagger$ \\
FFB & & - & & & & & $5.8^{*}$ & $5.5^{*}$ \\
ShS & 1.69 & & - & & $7.5 \ddagger$ & & $16.2_{\ddagger}^{\ddagger}$ & $21.6_{\ddagger}^{\ddagger}$ \\
MS & 1.55 & & & - & & $9.7 \ddagger$ & $20.4 \ddagger$ & $17.7_{\ddagger}$ \\
StS & 1.43 & & 0.68 & & - & $17.7_{\ddagger}^{\ddagger}$ & $30.2 \ddagger$ & $28.1^{\ddagger}$ \\
SP & 1.79 & & & 1.39 & 1.01 & - & $6.0^{*}$ & $11.7_{\ddagger}^{\ddagger}$ \\
MP & 1.87 & 1.11 & 0.87 & 1.18 & 1.35 & 0.51 & - & \\
LP & 1.91 & 1.04 & 1.14 & 0.79 & 1.53 & 0.84 & & - \\
\hline
\end{tabular}

Note -FF, free fall; FFB, free fall and bounce; ShS, shallow slope; MS, medium slope; StS, steep slope; SP, short pendulum; MP, medium pendulum; LP, long pendulum. Upper triangle: ${ }^{*} p<.05 ;{ }^{+} p<.01 ;{ }^{\ddagger} p<$ .001 and partial $F$ values. Lower triangle: differences in slope in proportion to mean slope.

and viewing distance constant, results in a nearly identical increase in optical velocities. Changes in either viewing distance or density alter the optical velocities in the same way. The resultant trajectories are so similar that it would be extremely difficult for an observer to resolve the difference between the two. Thus, in our displays, there was no information available to enable the observer to discriminate a change in ball mass from change in the size and viewing distance.

As already mentioned, the event-specific relations scaling time to spatial extents are approximations that ignore the effects of air resistance and friction. Variations in such effects that are produced by changes in material composition would have to be captured by adjustments in the event-specific scaling constants. We suggest that perception might be attuned to the approximate relations in part because they are parsimonious, but, more to the point, because visual information about the more complex, friction-specific circumstances may not be available, at least not in the event trajectories. Given this circumstance, one would not expect observers to be precisely tuned to the value of an event-specific scaling constant. Instead, given the use of the approximate scaling relations, the scaling constants would require adjustment depending on the masses and frictional forces operating on a given occasion.

As recently argued by Bingham and Pagano (1998), perception of definite spatial scale always entails calibration. Any measurement system is subject to fluctuations and to drift - that is, some instability. Furthermore, any measurement of definite distance requires scaling and calibration to the unit of expression (i.e., units for the control of reaching or walking, or units such as the meter for verbally expressed distances). Feedback is required for such calibration. Fortunately, feedback is generated with nearly every action. Every time one takes a step or reaches for an object, haptic and visual feedback is generated that can be used to calibrate the perception of definite spatial scale. So, given the ready availability of feedback and the inevitable instabilities of measurement systems, it would make sense to use simple, approximate scaling re- lations and to attune the scaling in local circumstances using feedback. The use of such approximate scaling relations would be smart (Runeson, 1977a) in the sense that the relations are simple and relatively general.

Experiment 2 was designed to test whether observers could accurately attune to the scaling constants given feedback and, if so, how rapidly. The strategy that we have described would entail fairly rapid attunement. In feedback trials, after the observer had used the pulley to position the patch-light marker ball at the perceived distance of the event, the ball was positioned at the correct distance by the observer under verbal guidance from the experimenter. Also, in Experiment 2, the observers were presented with event displays involving balls of identical size but of different densities (i.e., composed of different materials). As a result, the times in which the balls completed the events were significantly different. Five of the eight balls in the experiment were of a relatively low density. Feedback was provided only for a subset of these lowdensity balls. Thus, when viewing the higher density balls, the observers could interpret the change in trajectory as either a change in density or a change in size and viewing distance. Given the context of the task and the lack of any additional information about object density, we hypothesized that the observers would choose the latter. As a further test of the possibility that observers are sensitive to the relations among event-specific constants across events, we provided feedback for only a limited part of the range of distances for a single type of event. If observers correctly relate scaling across events, the effects of feedback should generalize to the other events.

\section{Method}

Observers. Eleven undergraduate and graduate students at Indiana University participated as observers. All had normal or corrected-tonormal vision. Two observers had participated in Experiment 1. The observers were paid $\$ 5$ per hour for $4 \mathrm{~h}$ of participation.

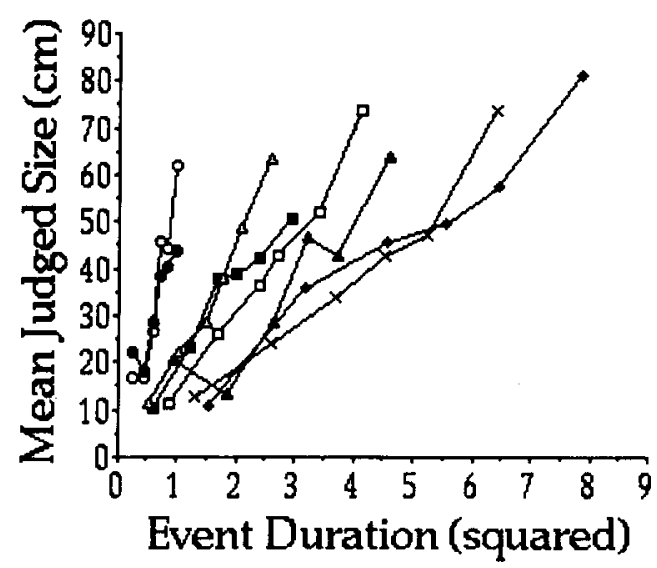

Figure 4. Mean judged size (in centimeters) regressed against event duration (sec) squared for all eight events: FF (open circles), FFB (filled circles), ShS (open squares), MS (filled squares), StS (open triangles), SP (filled triangles), MP (crosses), and LP (filled diamonds). 


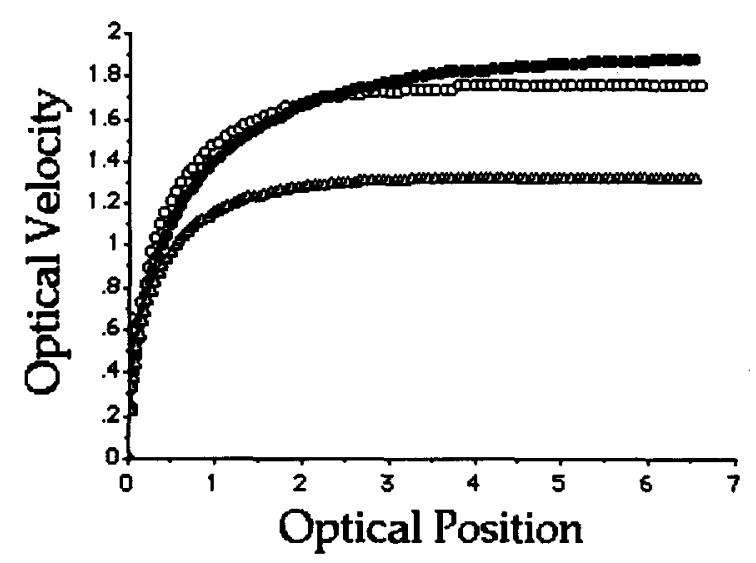

Figure 5. Optical phase-space portraits (created by plotting optical velocity against optical position) for a big and light ball (open triangles), a small and light ball (open circles), and a big and heavy ball (filled circles) rolling down an inclined plane. The small, light ball had the same density as the big, light ball.

Display generation. We filmed patch-light video displays of five different events: a ball in free fall (FF), a ball rolling down a shallow (ShS), medium (MS), and steep (StS) slope, and a ball on the end of a black rod swung as a pendulum (Pend). Eight balls of seven different sizes were filmed in each event at seven different distances (see Table 3) so as to preserve the same image size in each display. Each ball was painted a flat black and covered with a random placement of small pieces of reflective tape. The patches were cut from templates so that the relative shape and size of the patches were the same for all balls. The patches covered $17.9 \%$ of the surface area of each ball, were of negligible thickness, and adhered snugly to the ball's surface.

The events were filmed against a black background with a bright 1,000-W movie light positioned next to the camera shining on the event. The iris of the camera was adjusted to produce equivalent patch-light displays in all cases. In the FF events, the ball appeared unmoving at the top of the display and then dropped, falling beyond the edge of the display before striking the floor. The inclines were created by fixing two rigid pipes $(1.5 \mathrm{~cm}$ in diameter and $2 \mathrm{~m}$ in length) side by side and separated by small wooden blocks $(3 \mathrm{~cm}$ wide) at either end so as to form a track constraining the motion of the ball. The narrow track occluded only a negligible amount of the bottom portion of the larger balls. Furthermore, the track was covered with a black cloth that was pulled tight enough that the smaller, lighter balls rolled without depressing the cloth and thus were also not occluded by the track. With one end resting on the floor, the other end of the track was suspended from the ceiling by a cord adjusted to produce one of the three angles of inclination. The whole track was oriented in the frontoparallel plane with respect to the camera. In each display, the ball appeared unmoving at the top of the track and then rolled, finally rolling beyond the edge of the display before reaching the end of the track. For the Pend displays, each ball was attached to the end of a black wooden dowel that was suspended, in turn, from a hook. The pendulum appeared unmoving and displaced from the vertical by $40^{\circ}$ and then swung through two cycles before the display was terminated. The pendulum swung in the frontoparallel plane with respect to the camera. In all cases, only the ball was visible in the display appearing as a random set of patches.

The balls varied in material composition. We used three wooden balls, four styrofoam balls, and one air-filled plastic ball. For one size, there was both a wooden ball and a styrofoam ball. There was also one pair of wooden and styrofoam balls that differed in diameter by less than $1 \mathrm{~cm}$. The wooden balls were much heavier than their styrofoam counterparts. Table 3 describes the conditions in which a display of each ball was used.

The filmed events were digitized and arranged in random orders using Adobe Premiere 4.0 (1994) on a Macintosh Quadra A/V computer. We created two random orders of 24 trials each consisting only of StS event displays. In each random order, displays of all eight balls were presented three times each. These two random orders were used for the pretraining and posttraining blocks, respectively. In addition, we created a random order of 15 trials of the StS event, using displays of three midsize balls (see Table 3 ) presented five times each. This was used as the feedback block. A multievent random order of 50 trials, consisting of all five events and five ball distances (see Table 3), was used to test the generalization of training from the $\mathrm{StS}$ event to other events. In all cases, the event was repeated five times before the observer made a judgment.

Apparatus and Procedure. The observer wore a helmet equipped with a head-mounted monocular video display and camera. $\mathrm{He} / \mathrm{she}$ sat in a hallway. The walls and floor were covered with black cloth; the ceiling was hidden by a black cloth hung in front of and above the observer. A patch was placed over the observer's left eye. The eyepiece attached to the helmet was positioned over the right eye, allowing the observer to view a monochrome video display. The brightness and contrast of the monitor were adjusted to produce patch-light displays. The lens of the camera was attached to the helmet $9.0 \mathrm{~cm}$ to the right of the observer's right eye, pointing forward. The monocular field of view was $48^{\circ}$ (horizontal) $\times$ $39^{\circ}$ (vertical). We refer to the helmet and the attached lens as the headcam. The total weight of the headcam was $1.8 \mathrm{~kg}$.

A patch-light ball $12 \mathrm{~cm}$ in diameter was suspended from a pulley at the eye height of the observer. The pulley extended from just behind and to the right of the observer's head to the far end of the hallway at a distance of $8.7 \mathrm{~m}$. Only the ball was visible in the headcam display. The observers moved the ball by gripping and pulling the pulley cord with the right hand. The cord was successively grasped, pulled until the hand and arm were outstretched, released, grasped again next to the head, and pulled. The relation between

Table 3

Characteristics of the Eight Balls Used in Experiment 2 and the Sessions in Which They Were Used

\begin{tabular}{|c|c|c|c|c|c|}
\hline Ball & Material & Distance & Size & Mass & Session \\
\hline 1 & wood & 27.96 & 2.54 & 6.3 & pretraining and posttraining; multievent \\
\hline 2 & styrofoam & 60.96 & 5.1 & 8.6 & pretraining and posttraining; feedback \\
\hline 3 & wood & 86.36 & 7.6 & 133 & pretraining and posttraining \\
\hline 4 & styrofoam & 96.52 & 8.5 & 32.3 & pretraining and posttraining; multievent \\
\hline 5 & plastic & 152.40 & 11.4 & 128 & all four sessions \\
\hline 6 & wood & 193.04 & 15.2 & 1,000 & pretraining and posttraining \\
\hline 7 & styrofoam & 193.04 & 15.2 & 148 & all four sessions \\
\hline 8 & styrofoam & 259.08 & 22.8 & 349.1 & pretraining and posttraining; multievent \\
\hline
\end{tabular}




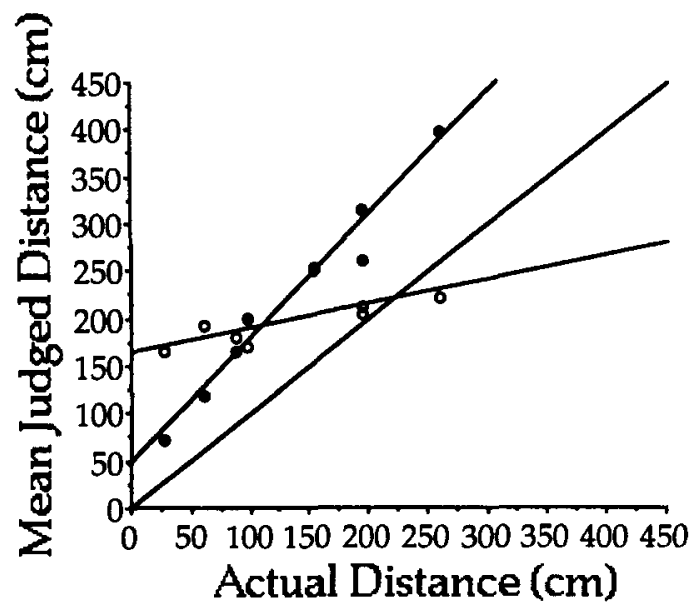

Figure 6. Best-fitting lines for mean judged distance (in centimeters) regressed against actual distance (in centimeters) for pretraining (open circles) and posttraining (filled circles).

the arm movements in moving the pulley and the expanding/contracting image of the ball as it moved toward or away from them enabled the observers to apprehend the distance of the ball. (A limited amount of distance information was also available from motion parallax resulting from head movement.) Essentially, distance was expressed in arm-length units.

The displays were presented to the observer via a VCR connected to the monitor of the headcam. While viewing a display, the observer was instructed to hold his or her head still and oriented as if looking down the hallway. After a display was shown, the monitor was switched to the head-mounted camera so that the observer could view the patch-light ball suspended from the pulley. At this point, the observer was free to make head movements in observing the marker ball. The observers were instructed to make distance judgments by moving the pulley until the marker ball was at a distance that matched the distance of the event in the display. Initially, the ball was located adjacent to the lens of the headcam for half of the observers and at the far end of the hallway for the remaining half. This was done to ensure that the observers were not using a limited range of sizes based on minimization of effort. After the observers made their judgment, the headcam monitor was switched off while an experimenter measured the distance of the ball from the lens of the headcam. The judgment apparatus allowed the observers to make distance judgments up to approximately $850 \mathrm{~cm}$.

On Day 1 of participation, the observers performed 24 trials judging the StS event displays (pretrain), followed by $15 \mathrm{StS}$ trials with feedback, and then a second set of 24 StS trials (posttrain). Feedback was guided verbally. After the distance judgment was recorded by the experimenter, the headcam display was switched on again so that the observer could see the marker while he or she was told to move the ball nearer or farther until the correct distance was located. Day 2 consisted of the 50-trial multievent block. Due to the weight of the headcam apparatus, the number of trials, and the effort required to make distance judgments, rest breaks were given periodically and at the request of the observer.

\section{Results}

This was a difficult task for 3 observers, who failed to understand the instructions and appeared to judge only image size (which, of course, was constant). The data from these observers were disregarded, and only the data of the 8 remaining observers were analyzed. There were
4 observers for whom the distance marker was placed near the head and 4 for whom the marker was placed at the far end of the hallway. We used a multiple regression to test for slope or intercept differences between these groups. For the pretraining session, the far-placement group had a higher intercept (206.7) than did the nearplacement group (86.3) (partial $F=18.8, p<.001$ ). However, for the posttraining session and for the Day 2 multievent session, there were no significant differences between these groups. The following analyses were performed on the combined data of the two groups.

Using the combined data of all observers, distance judgments from the pretraining session of Day 1 were regressed against actual distance. As shown in Figure 6, overall performance during pretraining was poor, with a slope of 0.26 , an intercept of 165.47 , and an $r^{2}$ of .02 . This low $r^{2}$ reflects both between- and within-subjects variability, as in Experiment 1, although the mean individual $r^{2}$ of $.25(S D=.28)$ was higher. For 3 observers, performance was especially poor, with slopes and $r^{2} \mathrm{~s}$ near zero and with extremely high intercepts.

For the training session, mean proportional errors were calculated for each trial. As shown in Figure 7, the observers appeared to adjust immediately, with a large error on Trial 1 , followed by a drop to less than one third of the level of mean error. Distance continued to be overestimated by about $0.5 \mathrm{~m}$ on average.

Distance judgments were also regressed against actual distance for the posttraining session of Day 1. Overall performance improved significantly from the pretraining block, with a slope of 1.33, an intercept of 44.04, and an $r^{2}$ of .54. Examination of individual performance revealed within-subjects consistency in this instance, with a mean individual $r^{2}$ of $.79(S D=.08)$. A multiple regression comparing performance for pretraining and posttraining was significant $\left[F(3,382)=54.1, p<.001, r^{2}=.3\right]$. The results of the multiple regression show that the posttraining slope was significantly higher than the pretraining slope (partial $F=48.8, p<.001$ ), and the intercept was

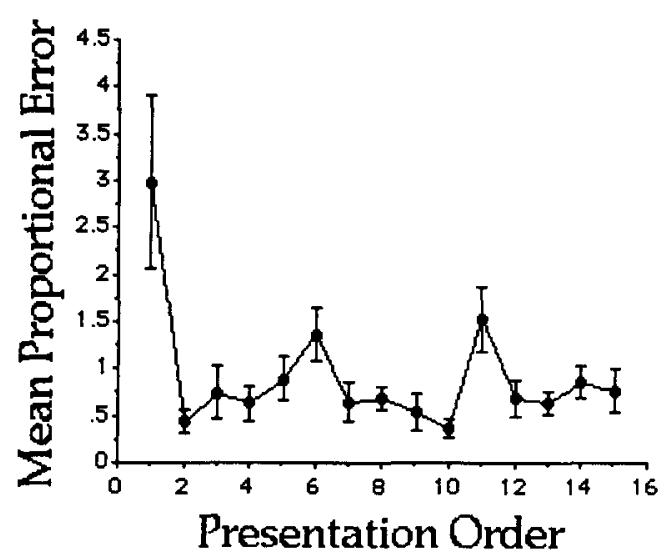

Figure 7. Mean proportional error (see text for explanation) against trial number for the training session. Error bars indicate standard error of the mean. 


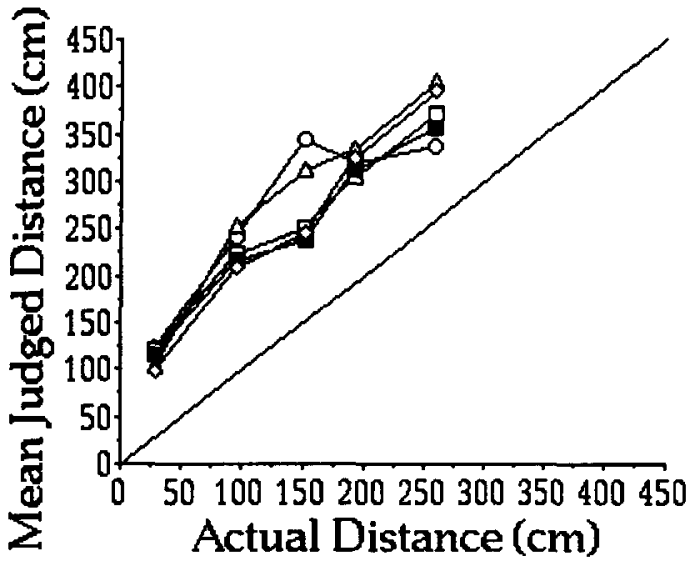

Figure 8. Mean distance judgments (in centimeters) for the multievent block of Experiment 2, Day 2, regressed against actual distance (in centimeters) for all five events: FF (open circles), ShS (open squares), MS (filled squares), StS (open triangles), and Pend (open diamonds).

significantly lower (partial $F=27.3, p<.001$ ). Figure 6 illustrates the improved performance after feedback.

The improved performance on Day 1 was stable over time. When actual distances were regressed on the judgments of the StS displays from the multievent block of Day 2 the result was a slope of 1.25 , an intercept of 101.4 , and an $r^{2}$ of .46 . A multiple regression comparing posttraining judgments (StS) to those of Day 2 StS displays yielded no significant differences for the slope or intercept. Performance for the other events from the multievent block was similar to that for the StS event, as shown in Figure 8. Multiple regressions using actual distance and comparing events two at a time revealed no significant differences in slope or intercept. As in Experiment 1, if the observers had simply been judging event durations, then this analysis should have yielded significant differences in slopes and a fan-shaped graph. Instead, the lines in the graph representing judgments of different events were collapsed together, indicating that the observers were judging distances.

As in Experiment 1, we examined the relation between event duration and judgments. A copy of the displays was made with a time code on each frame of each display, and event durations were measured directly from the displays. (Measurements of durations for the FF events were less reliable given the $30-\mathrm{Hz}$ video sampling rate [ $33 \mathrm{msec}$ per frame] and the extremely brief event durations $[\approx 450 \mathrm{msec}]$.) We compared events two at a time using multiple regression and regressing judgments on event durations. Out of 10 comparisons (five events taken two at a time), there were $9(90 \%)$ significant slope differences as shown in Table 4. As shown in Figure 9, these slope differences produced the fan effect for duration, as found in Experiment 1. Within events, distance judgments increased with duration (as did actual distance); however, across events, multiple durations gave rise to similar distance judgments, and any single duration gave rise to many different distance judgments.

The mass of the balls rolling down inclines affects the duration of the event significantly. We included in our events balls made of wood, styrofoam, and plastic (see Table 3 ). Ball mass varied as a function of material. This manipulation affected the observers' judgments. As shown in Table 3, Balls 3 and 4 were filmed at nearly the same distance but were made of different materials. The same was true of Balls 6 and 7. We compared judgments of distance for these four balls using a repeated measures analysis of variance, with distance and material as factors. The material factor had two levels (wood and styrofoam), as did the distance factor $(91.4$ and $193.0 \mathrm{~cm}$ ). (Balls 3 and 4 were treated as being at the same distance by taking a mean of their distance of $91.4 \mathrm{~cm}$.) There was a main effect for distance $[F(1,59)=65.5, p<.001]$. There was also a main effect for material $[F(1,59)=10.7$, $p<.01]$. The wooden balls were consistently judged as being closer than their styrofoam counterparts. The twoway interaction was significant $[F(1,59)=6.6, p<.05]$. The effect of material was stronger at the larger of the two distances. Figure 10 illustrates the effect.

To better elucidate what the observers were doing, we calculated the event-specific constants. We derived the physical constants by regressing actual distance against the square of event duration, as measured from the displays. The perceived constants were derived by regressing judged distance against the square of event duration from the multievent block data. Table 5 summarizes the results. A simple regression of physical on perceived constants was significant $\left(r^{2}=.99\right)$, with a slope $(=1.25)$ slightly greater than 1 and an intercept near zero, as shown in Figure 11. The perceived constants were similar to the physical values; thus, the observers appeared to attune to the event-specific constants available to them in the displays. Furthermore, they appeared to relate the value of the StS constant (for which they received feedback) to the constants for the other events. One way to see this is to compare the between-event ratios of the physical and perceived constants. If the observers related events appropriately, then the ratios between events for the physical and perceived constants should be equivalent. As shown in Table 6, the ratios are similar. A simple regres-

Table 4

Significant Slope Differences in Multiple Regressions of Event Duration on Judged Distance for Events Compared Two at a Time

\begin{tabular}{lccccc}
\hline & FF & ShS & MS & StS & Pend \\
\hline FF & - & $26.9_{+}^{+}$ & $21.1_{+}^{+}$ & $16.2_{+}^{+}$ & $31.7_{+}^{+}$ \\
ShS & 1.31 & - & & $7.3^{\dagger}$ & $8.1^{\dagger}$ \\
MS & 1.26 & & - & $4.1^{*}$ & $8.2^{+}$ \\
StS & 0.96 & 0.51 & 0.43 & - & $26.22_{+}^{+}$ \\
Pend & 1.61 & 0.63 & 0.71 & 1.06 & - \\
\hline
\end{tabular}

Note - FF, free fall; ShS, shallow slope; MS, medium slope; StS, steep slope; Pend, pendulum. Upper triangle: ${ }^{*} p<.05 ;{ }^{+} p<.01:{ }^{\ddagger} p<.001$ and partial $F$ values. Lower triangle: differences in slope in proportion to mean slope. 


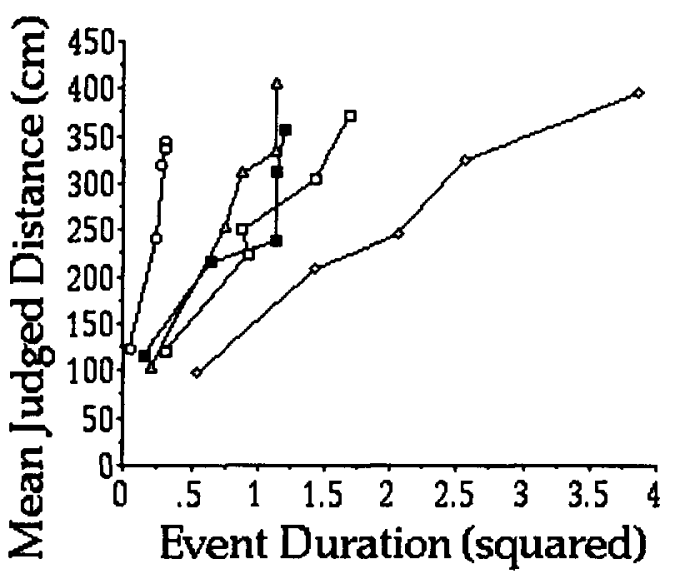

Figure 9. Mean judged distance (in centimeters) from the multievent block of Experiment 2, Day 2, regressed against event duration (in seconds) squared for all five events: FF (open circles), ShS (open squares), MS (filled squares), StS (open triangles), and Pend (open diamonds).

sion of empirical on observer values yielded an $r^{2}$ of .99 , a slope $(=0.96)$ near 1 , and an intercept near $0(=0.04)$. The smaller deviation from a slope of 1 in this instance indicates that perception of relative distance remained better than perception of definite distance despite the feedback training and resulting calibration.

It remained possible, however, that the observers did not generalize across events and that they merely adopted a range of values based on the initial feedback session and fit all subsequent judgments within this range. This would imply that the observers were not judging spatial scale but instead were able to identify five different durations within each event and place each into the appropriate response category. However, this would be a difficult task due to the completely randomized design of the multievent block and the fact that each event involved a different range of durations. Nevertheless, we performed an analysis to test for this possibility.

If the observers were identifying the durations and placing them into categories, we should expect to see an improvement in performance from the first time the observers were exposed to a given event/distance combination and the second time they saw that particular combination. (All combinations were presented twice.) We would expect this because of the difficulty in identifying the position within the order of the durations in each of the five events appearing throughout the random order (50 trials). We found no such improvement. Using the data from the multievent block, we performed simple regressions of actual distance against judged distance for each event for both the first and the second presentation of the event/distance combinations. The differences in slope, $y$-intercept, and $r^{2}$ values are shown in Table 7 . For all events except two (MS and Pend), the $r^{2}$ decreased. We performed a second analysis using just the data from the first presentation of each event/distance combination in the multievent block and comparing performance on the StS event (the training event) to all others. If the observers were using the above bias, we might expect that they should initially perform better on the StS event than on the others given the previous exposure and training. A multiple regression performed on these judgments, comparing each event with the StS event one at a time, revealed no significant differences in slope or intercept. As can be seen in Table 7, the StS first-occurrence $r^{2}$ was only slightly higher than all others. These analyses indicated that it is unlikely that the observers were making categorical judgments instead of truly judging distance, especially in light of the relationship between the physical and perceived constants, as shown in Figure 11.

\section{DISCUSSION}

Previous theoretical work has described how the trajectories of falling objects are scaled in proportion to their spatial scale (Saxberg, 1987a; Watson et al., 1992). Experimental work has provided a tentative reason to believe that observers are sensitive to this information and can use it to scale the visual field (Hecht et al., 1996; Muchisky \& Bingham, 1992; Pittenger, 1985, 1990; Saxberg, 1987b; Stappers \& Waller, 1993). The experiments reported here provide further empirical support that observers are sensitive to this relation between space and time. The large judgment variability of Experiment 1 indicates that definite scale might not have been perceived in these displays, which were impoverished compared with the information available about real events under normal viewing conditions. Nevertheless, judgments were better predicted by actual sizes than by mere durations, which, together with the event-specific trajectory forms, were the only information about spatial scale available in the displays. Thus, we concluded that the observers were sensitive to the nomological structure and spatial-temporal

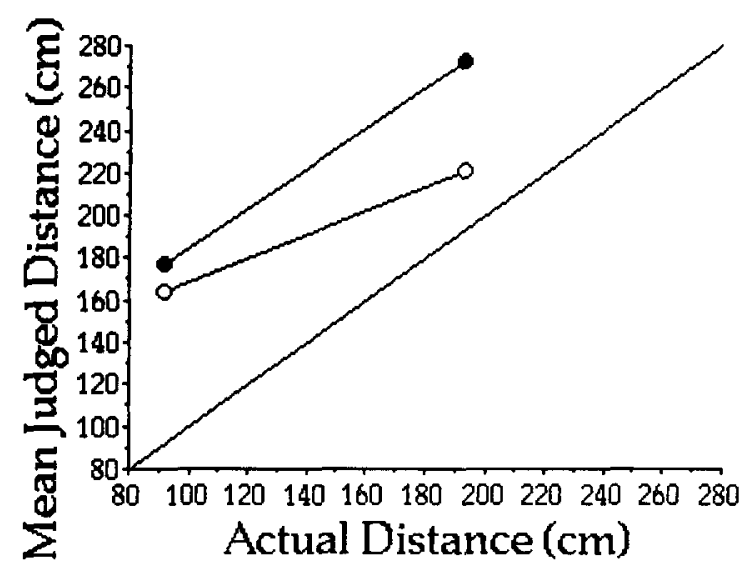

Figure 10. Mean judgments of wooden (open circles) and styrofoam (filled circles) balls at two distances. At each of the two distances were both a wooden and a styrofoam ball. Wooden balls were judged significantly closer. 


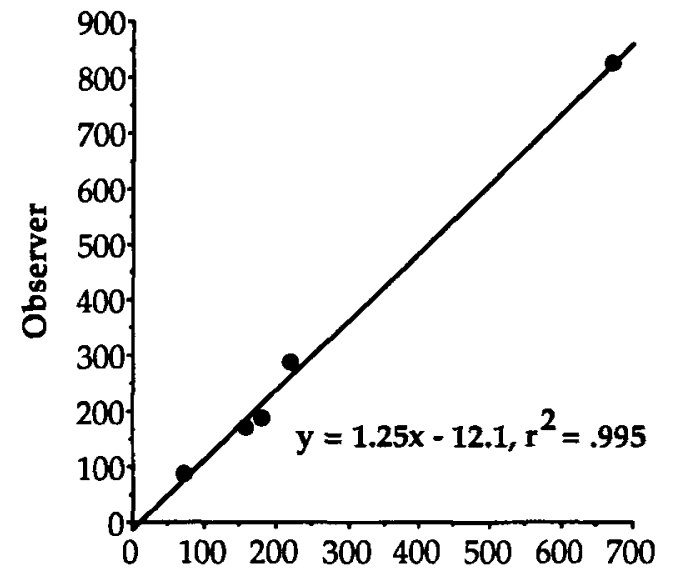

Figure 11. Mean observer constants regressed against the empirical constants. Observer constants were derived by calculating the slope of the regression line for judged size against the square of event duration for all 15 observers. Empirical constants were found by taking the slope of the regression line for actual size against the square of event duration.

form of events and to the relative relations among events, but they were not precisely tuned to the definite values of the scaling constants. The feedback in Experiment 2 helped the observers attune to the definite scale of the events. The observers calibrated quickly, in fact, in a single trial. This kind of performance might be expected if one were depending on local feedback to calibrate performance in specific tasks and situations. Given the hypothesized strategy, rapid response to feedback would be desirable. Once adjusted, furthermore, the calibration remained stable. The observers demonstrated remarkable improvement, which carried over to a 2 nd day. As illustrated in Table 5, the observers were using constants similar to the physically measured values. Furthermore, Table 6 shows that the observers correctly related the values of these constants across events, producing the same ratios that we derived directly from the displays. Further evidence that the observers appropriately scaled the relation between time and space was produced by analyses of the simple correlation between event durations and judgments. There was no single monotonic relation between duration and perceived spatial scale. Instead, the observers exhibited a different relation for each event. This pattern of results was found in the multievent blocks of Experiments 1 and 2. We conclude that observers are sensitive to the form of the relation between time and space but are not initially tuned to the precise values of the constants, at least when viewing the types of displays used in Experiments 1 and 2. A small amount of feedback was sufficient to provide the observers with the scaling constants (assuming constant mass density of different objects in the different events).

However, even after feedback, performance was different for same-sized balls of different material. Specifically, heavy balls were judged to be closer than lighter balls of the same size. Thus, attunement to the values of the scaling constants does not appear to be robust in the face of perturbations of mass density. Mass variations alter trajectories quantitatively because they alter the effects of friction and air resistance on the object (and because, in rolling events, the mass distribution determines the inertial resistance to rotational acceleration). As discussed by Hecht et al. (1996), variations in these forces complicate matters for perception, and they led these authors to conclude that trajectory information cannot be used optimally when viewing objects affected by such perturbations. However, within a specific set of objects (e.g., balls of the same density), our observers performed very well, even though these objects were subject to forces of friction and air resistance. Our observers, however, did not detect manipulations of mass density and, thus, were less accurate when judging the heavier balls. Optimal performance appears to require only that the objects do not vary in mass (unless there is additional information to that effect), but not an absence of friction or air resistance. With regard to variations in mass density, we note that homogeneity of mass density is common to many situations and tasks, and especially with a historical or evolutionary view, tasks involving the use of preindustrial materials - that is, stone, wood, clay, or water.

Hecht et al. (1996) also pointed out that observers could have trouble using trajectory information given research demonstrating poor performance at judging differences in acceleration (Babler \& Dannemiller, 1993; Calderone \& Kaiser, 1989; Rosenbaum, 1975). Even though gravitational acceleration is a component of the scaling between space and time, our analysis suggests that observers need only know the value of the scaling constant, and they need not perceive accelerations per se. However, to use the appropriate scaling constant, an observer must first identify the event. Event identification may entail recognition via characteristic trajectory forms (Bingham, 1995; Bingham et al., 1995; Muchisky, 1995), forms that appear in the phase portrait of an event (e.g., see Figure 5). While acceleration can be used to describe the form of a trajectory, it need not be used in preference to the mere description of velocity and its variation over time and positions in an event. Observers do not need to be able to discern differences in acceleration in order to recognize an event via trajectory forms. They only need to be able to detect the relevant variations in velocity. Muchisky (1997) has shown that human observers are in

Table 5

Physical and Perceived Event-Specific Scaling Constants

\begin{tabular}{lcc}
\hline Event & Physical & Perceived \\
\hline FF & 671.11 & 824.46 \\
StS & 219.81 & 289.47 \\
MS & 181.49 & 187.49 \\
ShS & 159.31 & 171.53 \\
Pend & 70.53 & 89.28 \\
\hline
\end{tabular}

Note-FF, free fall; StS, steep slope: MS, medium slope; ShS, shallow slope; Pend, pendulum. 
Table 6

Ratios Between Events for the Scaling Constants Shown in Table 5

\begin{tabular}{lcc}
\hline Event & Physical & Perceived \\
\hline StS-MS & 1.21 & 1.54 \\
StS-ShS & 1.38 & 1.69 \\
StS-FF & 0.33 & 0.35 \\
StS-Pend & 3.12 & 3.24 \\
MS-ShS & 1.14 & 1.09 \\
MS-FF & 0.27 & 0.23 \\
MS-Pend & 2.57 & 2.10 \\
ShS-FF & 0.24 & 0.21 \\
ShS-Pend & 2.26 & 1.92 \\
FF-Pend & 9.52 & 9.23 \\
\hline
\end{tabular}

Note--StS, steep slope; MS, medium slope; ShS, shallow slope; FF, free fall; Pend, pendulum.

fact quite sensitive to variations in trajectory form (see also Muchisky, 1995).

When viewing real events under normal viewing conditions, information about the actual value of the scaling constants could come from a variety of sources. For example, Bingham (1993a) found that observers could use the scale derived from apprehension of tree forms to apprehend the size of other objects in the visual field - that is, to confer a definite spatial scale on the whole field. Thus, once the scale of any given object in the visual field is known, an observer could potentially relate its scale to an event, such as a falling object. Interaction with the objects could provide haptic feedback, which could also inform the observer about the definite scale of the event. Furthermore, sensitivity to the interrelated dynamical structures of different events allows observers to relate the scaling of one event to many others. The point is that we inhabit a world of regularities, both in the structure of surfaces and layout and in the structure of events. Our visual system is sensitive to these diverse but coherent regularities. On a breezy autumn day, one may look out on a scene populated by falling leaves and swaying treesthat is, free fall and pendulum events. The gradients formed by the distributions of these events in the surroundings are as informative and as relevant to perception as the parallax and texture gradients more familiar to vision researchers. The fact, as shown by these exper-

Table 7

Simple Regressions of the First and Second Occurrences of Each Event in the Multievent Block of Experiment 2, Day 2

\begin{tabular}{lllcl}
\hline Event & Occurrence & Slope & $y$-intercept & $r^{2}$ \\
\hline FF & First & 1.03 & 89.2 & .40 \\
& Second & 0.84 & 183.0 & .21 \\
ShS & First & 1.11 & 107.7 & .44 \\
& Second & 0.92 & 99.6 & .35 \\
MS & First & 1.07 & 126.1 & .31 \\
& Second & 0.99 & 69.5 & .44 \\
StS & First & 1.34 & 95.5 & .48 \\
& Second & 1.16 & 106 & .44 \\
\multirow{2}{*}{ Pend } & First & 1.17 & 75.7 & .43 \\
& Second & 1.36 & 63.4 & .48 \\
\hline
\end{tabular}

Note-FF, free fall; ShS, shallow slope; MS, medium slope; StS, steep slope; Pend, pendulum. iments, that the visual system is sensitive to event structures and to the relation among events provides a powerful and efficient way to achieve definite spatial scaling of the visual surround.

\section{REFERENCES}

Adobe Premiere 4.o [Computer Software] (1994). Mountain View, CA: Adobe Systems.

Babler, T. G., \& DANNEMiller, J. L. (1993). Role of image acceleration in judging landing location of free-falling projectiles. Journal of Experimental Psychology: Human Perception \& Performance, 19, 15-31.

Bingham, G. P. (1987). Kinematic form and scaling: Further investigations and the visual perception of lifted weight. Journal of Experimental Psychology: Human Perception \& Performance, 13, 155-177.

Bingham, G. P. (1993a). Form as information about scale: Perceiving the size of trees. Journal of Experimental Psychology: Human Perception \& Performance, 19, 1139-1161.

BINGHAM, G. P. (1993b). Scaling judgments of lifted weight: Lifter size and the role of the standard. Ecological Psychology, 5, 31-64.

Bingham, G. P. (1995). Dynamics and the problem of visual event recognition. In R. F. Port \& T. van Gelder (Eds.), Mind as motion: Explorations in the dynamics of cognition (pp. 403-448). Cambridge, MA: MIT Press.

Bingham, G. P., \& Pagano, C. C. (1998). The necessity of a perception-action approach to definite distance perception to guide reaching. Journal of Experimental Psychology: Human Perception \& Performance, 24, 1-25.

Bingham, G. P., Rosenblum, L. D., \& Schmidt, R. C. (1995). Dynamics and the orientation of kinematic forms in visual event recognition. Journal of Experimental Psychology: Human Perception \& Performance, 21, 1473-1493.

CAlderone, J. B., \& Kaiser, M. K. (1989). Visual acceleration detection: Effect of sign and motion orientation. Perception \& Psychophysics, 45, 391-394.

Eby, D. W., \& Braunstein, M. L. (1995). The perceptual flattening of three-dimensional scenes enclosed by a frame. Perception, 24, 981-993.

EPSTEIN, W. (1967). Varieties of perceptual learning. New York: McGrawHill.

Hecht, H., Kaiser, M. K., \& Banks, M. S. (1996). Gravitational acceleration as a cue for absolute size and distance? Perception \& Psychophysics, 58, 1066-1075.

InTERACTIVe Physics II [Computer Software] (1992). San Francisco: Knowledge Revolution.

MacroMind Accelerator [Computer Software] (1989). San Francisco: MacroMind.

MUCHISKY, M. M. (1995). Event identification via dynamically governed trajectory forms. In B. G. Bardy, R. J. Bootsma, \& Y. Guiard (Eds.), Studies in perception and action 1/l: Eighth International Conference on Perception and Action (pp. 359-362). Hillsdale. NJ: Erlbaum.

MUCHISKY, M. M. (1997). Trajectory forms as visual information in bounce events. In M. A. Schmuckler \& J. M. Kennedy (Eds.), Studies in perception and action IV: Ninth International Conference on Perception and Action (pp. 43-46). Hillsdale, NJ: Erlbaum.

Muchisky, M. M., \& Bingham, G. P. (1992). Perceiving size in events via kinematic form. In J. Kruschke (Ed.), Proceedings of the 14th Annual Conference of the Cognitive Science Society (pp. 1002-1007). Hillsdale, NJ: Erlbaum.

Pittenger, J. B. (1985). Estimation of pendulum length from information in motion. Perception, 14, 247-256.

Pittenger, J. B. (1990). Detection of violations of the law of pendulum motion: Observers' sensitivity to the relation between period and length. Ecological Psychology, 2, 55-81.

Poulton, E. C. (1989). Bias in quantifying judgments. Hillsdale, NJ: Erlbaum.

Rosenbaum, D. A. (1975). Perception and extrapolation of velocity and acceleration. Journal of Experimental Psycholog:: Human Perception \& Performance, 1, 395-403. 
RUNESON, S. (1977a). On the possibility of "smart" perceptual mechanisms. Scandinavian Journal of Psychology, 18, 172-179.

RUNESON, S. (1977b). On the visual perception of dynamic events (Acta Universitatis Upsaliensis: Studia Psychologica Upsaliensia 9). Stockholm: Almqvist \& Wiksell. (Reprinted 1983)

RUNESON, S. (1994). Perception of biological motion: The KSD principle and the implications of the distal versus proximal approach. In G. Jansson, S. S. Bergstrom, \& W. Epstein (Eds.), Perceiving events and objects (pp. 383-405). Hillsdale, NJ: Erlbaum.

RUNeson, S., \& Frvkholm, G. (1981). Visual perception of lifted weight. Journal of Experimental Psychology: Human Perception \& Performance, 7, 733-740.

RUNESON, S., \& FrYkHOLM, G. (1983). Kinematic specification of dynamics as an informational basis for person and action perception: Expectation. gender recognition, and deceptive intention. Journal of Experimental Psychology: General, 112, 585-615.

RUNESON, S., \& VEDELER, D. (1993). The indispensability of precolli- sion kinematics in the visual perception of relative mass. Perception \& Psychophysics, 53, 617-632.

SaXberG, B. V. H. (1987a). Projected free fall trajectories: I. Theory and simulation. Biological Cybernetics, 56, 159-175.

SaxBerg, B. V. H. (1987b). Projected free fall trajectories: II. Human experiments. Biological Cybernetics, 56, 177-184.

Stappers, P. J., \& Waller. P. E. (1993). Using the free fall of objects under gravity for visual depth estimation. Bulletin of the Psychonomic Society, 31, 125-127.

TODD, J. T., \& WARREN, W. H. (1982). Visual perception of relative mass in dynamic events. Perception, 11, 325-335.

Watson, J. S., Banks, M. S., von Hofsten, C., \& Royden, C. S. (1992). Gravity as a monocular cue for perception of absolute distance and/or absolute size. Perception, 21, 69-76.

(Manuscript received January 23, 1997; revision accepted for publication September 24, 1997.) 